\title{
De la passivité à la collaboration : l'évolution des relations entre cacaoculteurs et industriel en Équateur*
}

\author{
Michel Dulcire \\ Cirad \\ Environnement et Société \\ UMR Innovation \\ BP 5032 \\ TA $60 / 15$ \\ 73, rue J.-F. Breton \\ 34398 Montpellier cedex 5 \\ <michel.dulcire@cirad.fr>
}

\begin{abstract}
Résumé
Les relations entre agriculteurs et industriels sont toujours asymétriques, et sont source de difficultés pour développer des activités conjointes. Un industriel chocolatier français, fortement motivé par le potentiel aromatique d'un cacao d'Équateur, a proposé un contrat à une organisation de producteurs de ce pays. Il s'est assuré des services d'un chercheur, expert en cacaos aromatiques, comme médiateur au niveau technique et organisationnel, pour réussir la requalification commerciale de cette variété historique. L'objet de notre étude était de comprendre comment et en quoi le partenariat entre le chercheur, l'industriel et les producteurs avait fait évoluer les modes relationnels et contractuels entre cet industriel et les associations de producteurs. Nous insistons dans cet article sur la façon dont se sont établies des relations entre l'industriel et l'organisation des producteurs, sur la base de ce contrat. Les producteurs s'engagent dans le partenariat imposé par l'acheteur pour des raisons économiques de valorisation de leur activité productive de leur cacao traditionnel. La négociation initiale du cahier des charges reste donc passive. Puis les producteurs entrent peu à peu dans ce processus de requalification territoriale. Ils deviennent partenaires actifs de leur acheteur et font évoluer les termes du contrat par une négociation intégratrice. En conclusion, ces apprentissages techniques et organisationnels restent cependant incomplets, et n'ont pas encore stabilisé une alliance qui reste fragile.
\end{abstract}

Mots clés : apprentissage ; approche participative ; cacao ; contrats ; Equateur ; partenariat.

Thèmes : économie et développement rural ; productions végétales ; transformation, commercialisation.

\section{Abstract \\ From passivity to collaboration: Changes in the links between cocoa farmers and a manufacturer in Ecuador}

The relationship between farmers and manufacturers are always asymmetric, making it difficult to propose joint activities. A French chocolate manufacturer, strongly motivated by the potential aroma of cocoa in Ecuador, proposed a contract to an organization of producers in this country. He enlisted the services of an investigator, a technical and organizational expert in aromatic cocoas, to successfully carry out the commercial regeneration of this historical variety. The purpose of our study was to understand how and why the partnership between the researcher and the industrial producers needed to develop means of negotiating and contractual relationships between the industry and producer associations. We emphasize in this article how a stable relationship between these two actors was established on the basis of this contract. The producers entered the partnership with the purchaser only for economic reasons, to make the production of their traditional cocoa more attractive. During the initial negotiation of the specification they remained quite passive. The producers then gradually integrated the process of territorial re-qualification. They have now become active partners of the industrialist and are developing the terms

\footnotetext{
* Pour citer cet article: Dulcire M. L'évolution des relations entre cacaoculteurs et industriel en Équateur: de la passivité à la collaboration. Cah Agric 2010; 19 : 249-54. Doi : 10.1684/ agr.2010.0395
} 
of the contract through an inclusive negotiation. In conclusion, we show that the technical and organisational learning remains incomplete and have not yet stabilized an alliance. The relationship thus remains fragile.

Key words: cocoa; contracts; Ecuador; learning; participatory approaches; partnerships.

Subjects: economy and rural development; processing, marketing; vegetal productions.

$\mathrm{H}$ istoriquement, dans la zone côtière de l'Équateur, une variété endémique de cacaoyers nommée "nacional" produit un cacao aux qualités reconnues typées (au sens de Salette, 1985). Ce cacao a aussi une valeur sentimentale pour les Équatoriens, puisque ses ventes en contrebande ont aidé à financer la guerre d'indépendance contre la couronne d'Espagne, qui, en outre, ne l'achetait qu'à un prix dérisoire aux producteurs. Ensuite, les exportations massives à la fin du XIX et au début du $\mathrm{xx}^{\mathrm{e}}$ siècles de la "pepa de oro " réalisées par de grands propriétaires terriens ont propulsé l'Équateur au premier rang des producteurs mondiaux. L'arrivée massive de maladies, comme la moniliose ou le balai de sorcière, et la première guerre mondiale ont mis fin à cette époque "dorée ". Par la suite, sous la double influence de la réforme agraire et de la reconversion des grandes plantations de cacaoyers en bananeraies, la production a été assurée par de petits producteurs, qui revendiquent aujourd'hui comme historique et culturel leur "cacao nacional", dont la production est néanmoins en baisse continue, en volume et en qualité.

C'est en réponse à cette évolution régressive qu'une intervention conjointe de l'Union européenne (UE) et du ministère de l'Agriculture et de l'Élevage (Ministerio de Agricultura y Ganaderia [MAG]) est réalisée de 1995 à 2000. La recherche, responsable de son déroulement, devait répondre à une attente de résultats techniques et scientifiques sur les maladies du cacaoyer, ainsi que sur ses qualités organoleptiques, mais aussi d'actions concrètes sur le court terme.

Dès le début, des responsables d'organisations paysannes, malheureusement peu structurées, se mobiliseront pour que cette intervention sous forme de "projet " réponde aux aspirations des producteurs. C'est ainsi qu'un des chercheurs de l'équipe française interviendra hors station et en association avec les produc- teurs: expert en cacao, il travaillera à la mise en place d'une capacité locale d'évaluation organoleptique du traitement postrécolte du cacao et au renforcement du fonctionnement collectif. Les résultats seront cependant inégaux : les tentatives de réhabilitation des vieilles plantations sont un échec, la structure de vulgarisation agricole ne survit pas à l'arrêt du financement et l'organisation paysanne sera mise en faillite. En revanche, la collection de cacaoyers aromatiques locaux constitue une première mondiale, une capacité d'évaluation organoleptique est acquise, et ce chercheur a gagné la confiance des producteurs et de leurs associations qui le sollicitent pour des appuis techniques et organisationnels.

C'est à la suite de cette intervention publique, peu convaincante, qu'entre en scène un industriel chocolatier français spécialisé dans le cacao aromatique, désireux de diversifier ses sources d'approvisionnement. Ses attentes portent explicitement sur des cacaos de qualité originale, produits en agriculture biologique, exigences qu'il reporte dans un " contrat équitable ". Il exige par ailleurs, pour réduire les coûts d'intermédiation, de s'engager avec une organisation de producteurs et non avec des individus. Après une étude de faisabilité commandée à ce chercheur, il décidera de passer un contrat avec la coopérative Unocace (Unión Nacional de las Organizaciones Campesinas Cacaoteras de Ecuador) pour lancer une opération durable, labellisée "bio-équitable ". C'est à la demande de l'industriel que ce chercheur, expert en cacao, connu et reconnu par les producteurs, en deviendra partenaire.

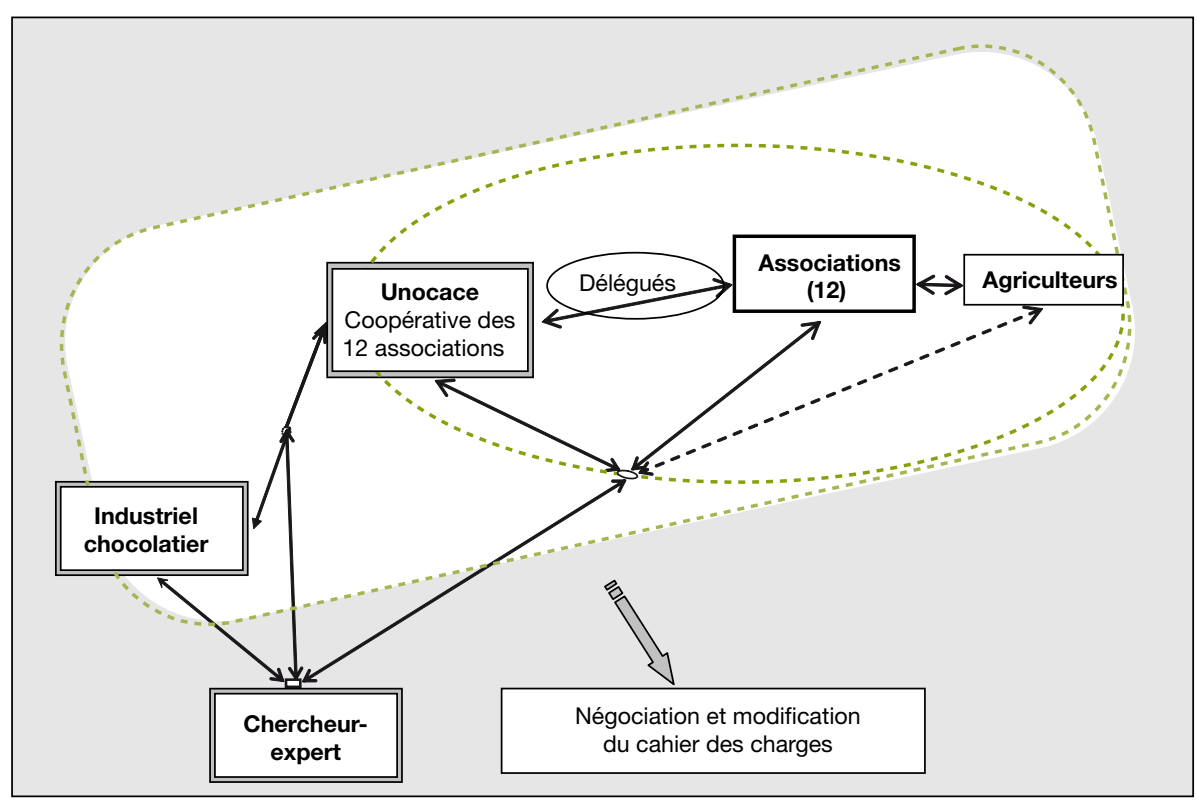

Figure 1. Relations entre l'industriel, les producteurs et leurs organisations, et le chercheur.

Figure 1. Relations between the industrialist, the farmers and their organisations, and the researcher. Unocace : Unión Nacional de las Organizaciones Campesinas Cacaoteras de Ecuador. 
Notre étude se situait dans le cadre d'un projet sur la recherche en partenariat et s'est intéressée au processus de traduction technique et organisationnelle de cette demande industrielle, une demande soumise à des organisations de producteurs, avec l'intervention d'un chercheur (figure 1). Notre hypothèse était que le respect des règles de fonctionnement imposées par l'industriel a amélioré la compétence technique et organisationnelle des coopérateurs, ainsi que leur capacité de négociation collective des termes du contrat. Nous avons conduit des entretiens compréhensifs avec différents acteurs concernés: agriculteurs et leurs organisations, industriel, chercheurs, techniciens; mais aussi avec des acteurs non concernés (encadré 1). Nous analysons dans cet article la façon dont les relations entre l'industriel et les agriculteurs ont évolué, leurs conséquences au niveau technique et organisationnel, ainsi que les rôles du chercheur-expert dans ce processus. Enfin, nous posons la question de l'autonomie effective des producteurs comme produit de ces apprentissages.
La construction d'un système relationnel entre les partenaires du contrat

\section{Quatre acteurs partenaires, quatre attentes}

Un industriel importateur

qui a besoin d'un partenaire collectif

L'entreprise chocolatière est à la recherche de cacaos fins et typés, issus de l'agriculture biologique, afin d'augmenter ses volumes et de diversifier ses sources d'approvisionnement. Le potentiel qualitatif du cacao "nacional " équatorien l'intéresse, bien qu'il soit en général pénalisé sur les marchés par ses nombreux défauts, dus en particulier à un traitement postrécolte inadéquat.

Cet opérateur ne veut s'engager qu'avec des groupements organisés (figure 1) et non avec des individus isolés, et cela selon les modalités du commerce équitable. Il exige que tous les engagements réciproques figurent dans un contrat, afin de "favoriser le lien social et humain" et d'en assurer la pérennité. Conscient de la complexité de la situation, il s'assure des services du chercheur expert en cacao qui connaît les producteurs et qui partage ses convictions.

\section{Des producteurs \\ qui veulent devenir exportateurs et s'organisent en conséquence}

Les coopératives de base ont mis en place avec l'appui du projet de l'UE une organisation faitière, Unocace. Leur attente devient alors : en première analyse, vendre leur production à un prix stable le meilleur possible et, surtout, le plus vite possible après la récolte, pour améliorer leur revenu. Mais ils sont aussi en quête d'une certaine reconnaissance sociale de leur action pour une culture traditionnelle emblématique.

Les plus progressistes savent qu'il faut alors saisir l'offre de l'industriel. Mais le mode contractuel qu'il exige les oblige à s'organiser et à s'impliquer activement. Leurs habitudes sont bousculées, ils n'ont pas d'autre choix.

\section{Encadré 1 \\ Méthode de travail}

Nous avons travaillé essentiellement par entretien avec différents acteurs engagés dans l'opération. Ces " entretiens compréhensifs" (Kaufmann, 2005) ont été individuels et collectifs, et réalisés dans les lieux de travail des acteurs : ils étaient articulés sur l'histoire individuelle et collective, le fonctionnement actuel, et les attentes de ces acteurs pour le futur.

Vingt-huit enquêtes ont été effectuées.

\section{Quinze (15) enquêtes collectives}

- Des groupes d'agriculteurs (12) au sein des associations, de 2 à 13 participants, et avec ou sans leurs délégués ;

- l'équipe technique de la coopérative Unocace (Unión Nacional de las Organizaciones Campesinas Cacaoteras de Ecuador);

- deux Organisations non gouvernementales (ONG), acteurs non concernés, qui organisent des formations collectives pour les agriculteurs de ce contrat et appuient la construction de structures collectives de traitement postrécolte.

\section{Treize (13) enquêtes individuelles}

- Des agriculteurs de base et des délégués (6);

- les deux techniciens et le responsable, salariés de Unocace ;

- l'industriel ;

- le chercheur français ;

- des chercheurs sur le cacao équatoriens (2), directeurs successifs de la station de recherche, qui ont été partie prenante du volet sélection et d'évaluation organoleptique.

Il faut souligner le fait que les agriculteurs ont été beaucoup plus réactifs en entretiens de groupe qu'en entretien individuel. Ces entretiens collectifs ont rapidement débouché sur des discussions ouvertes et soutenues entre les participants, et cela indépendamment de notre présence. Ces débats autonomes ont porté spontanément sur leurs lectures personnelles et collectives de leur organisation, tant de son histoire que de son fonctionnement actuel et des avenirs. Nos questions ont, dans ces cas, porté sur des précisions et, parfois, relancé un sujet important.

Plusieurs agriculteurs seront enquêtés deux fois : certains en individuel et en collectif; d'autres au sein de leur association et en dehors, lors d'un atelier interne des délégués, auquel cas ils ne sont comptés qu'une fois. 


\section{Une organisation déstabilisée}

Un meilleur prix d'achat du cacao avait été, selon le président d'Unocace, la raison principale des producteurs pour s'organiser dans le cadre du projet européen, mais les intermédiaires n'ont jamais proposé un meilleur prix pour les inciter à faire de la qualité.

L'arrêt du projet a déstabilisé l'Unocace, compte tenu de sa faible expérience et de l'absence de contrats pour la vente du cacao. Créée pour le marché, elle n'a pas su s'y maintenir et elle a fortement décapitalisé. Cependant, les producteurs n'expriment pas de déception après cet échec, comme c'est souvent le cas lorsque des résultats sur le court terme ne sont pas au rendez-vous (Faure et al., 2007). C'est d'ailleurs leur volonté de continuer qui incitera l'industriel chocolatier à aider Unocace à se renforcer comme organisation faitière, comme acteur partenaire de l'élaboration d'un contrat de commerce équitable.

\section{Un chercheur écartelé}

Il est d'une part déjà reconnu comme un allié par les producteurs, qui le connaissent et l'écoutent. Son rôle de conseiller technique de l'industriel l'amène d'autre part à répondre d'abord à ses exigences, en médiateur et formateur vis-à-vis des producteurs, de leurs associations de premier niveau et de la coopérative (figure 1). Ces deux classes d'acteurs n'ont pas les mêmes intérêts ni le même point de vue, et ils ne travaillent pas au même rythme. Il doit donc permettre à ces parties de prendre la parole, et intervenir tant par ses recherches ou expertises dans le domaine de la production du cacao que par ses conseils sur le fonctionnement technique et organisationnel de cette filière "bio et équitable ".

Le risque de "dispersion excessive " (Thill, 2001) de ses activités, entre connaissance scientifique, action et apprentissages collectifs et individuels (Freire, 1974), renvoie au risque de non-reconnaissance par l'institution de recherche.

\section{Le contrat, des engagements réciproques}

L'industriel affirme que tous les acteurs doivent pouvoir tirer profit du contrat, dont il a proposé un modèle dès le début, et que leur adhésion active est indispensable à sa pérennité. Mais le mode d'organisation des producteurs était alors balbutiant. Le contrat initial de l'industriel a donc de fait imposé ses exigences. L'implication des producteurs est progressive : leurs pratiques améliorent le fonctionnement de leurs associations et leur donnent les moyens d'exprimer des demandes à l'industriel. Le contrat actuel représente un " consensus interculturel ", donc le produit d'une "construction négociée " (Denoux, 2006) entre l'industriel et les producteurs, aux stratégies différentes, et appuyée par le chercheur (figure 1). Ce contrat liste les responsabilités et les droits des trois parties : celles des signataires, d'une part - l'industriel et la coopérative faîtière -, et celles des associations, d'autre part, qui sont sous le contrôle de la coopérative. Le cahier des charges (tableau 1) en explicite les conditions et responsabilités pour chacune de ces trois parties : par exemple la variété traditionnelle, les modalités économiques et d'investissement, l'assistance technique, les modes de fonctionnement organisationnel, etc., et, surtout, les tâches de contrôle des types et modes d'itinéraires techniques (culturaux et postrécolte), c'est-à-dire un ensemble de facteurs dont la maîtrise joue sur la stabilité du contrat (Akrich, 2006).

Les producteurs se sont ainsi lancés dans l'aventure "cacao bio et équitable ", avec les exigences de qualité de production que cela implique. Cependant, la grande majorité d'entre eux n'a pas ressenti l'attente, de la part de l'industriel, d'une participation active des producteurs. La première version du contrat a donc donné lieu à peu d'échanges. Les agriculteurs qualifient spontanément leur ralliement initial au contrat comme une contrainte subie, en "l'absence d'autre choix " ou "faute de mieux ", ou encore comme une opportunité, celle des prix attractifs proposés. C'est en cours d'entretien, et en discussion collective, que leur implication progressive dans la dynamique contractuelle est énoncée. Cette situation illustre la difficulté qu'ont eue les producteurs à commencer à se coordonner entre eux pour élaborer une intention commune. Mais elle montre aussi l'importance d'un leader dans la construction du système relationnel, pour surmonter les difficultés et faire avancer le processus.

\section{Du vieux monde}

\section{au nouveau monde: s'organiser pour survivre}

\section{L'organisation, un acteur imposé par l'industriel}

Wyvekens (2003) souligne que les bonnes pratiques sont des pratiques efficientes, mais qui sont aussi éthiquement honnêtes. Elles changent quelque chose dans les modes de travail, techniques et relationnels, de façon durable, tant socialement que culturellement. La demande du chocolatier a représenté une opportunité, une "arène d'intercompréhension "

\section{Tableau 1. Extraits du cahier des charges des trois parties.}

Table 1. Extracts from the specification involving the 3 parties.

\begin{tabular}{|c|c|c|}
\hline Industriel chocolatier & Associations de base & Unocace \\
\hline Assistance technique & Variété traditionnelle & \multirow[t]{2}{*}{ Contrôle et traçabilité du produit } \\
\hline Coût certification & $\begin{array}{l}\text { Respect règlement agriculture biologique } \\
\text { par contrôle interne }\end{array}$ & \\
\hline Achat du cacao à Unocace & Achat fèves fraîches aux adhérents & Collecte coopératives \\
\hline & Travail collectif postrécolte & Conditionnement \\
\hline Préfinancement des achats sans intérêt & Paiement au producteur en livraison & Gestion exportations \\
\hline Appui infrastructures & Construction infrastructures & Coordination infrastructures \\
\hline
\end{tabular}

Unocace : Unión Nacional de las Organizaciones Campesinas Cacaoteras de Ecuador. 
(Fixmer et Brassac, 2004) fonctionnelle entre la coopérative et les associations de producteurs d'un côté, et l'industriel de l'autre. La coopérative Unocace est la signataire désignée du contrat et le développement de son pouvoir d'agir est ici supérieur à celui de l'individu. Son assemblée, composée des délégués élus des associations, décline le cahier des charges en objectifs et en actions collectives, pour tous (tableau 1), en fixe les modes de contrôle et agit indépendamment des demandes individuelles. En ce sens, l'engagement du collectif à respecter le cahier des charges bio-équitable est à la fois une contrainte extérieure imposée aux individus et un facteur de valorisation de ce travail.

L'innovation, au fur et à mesure qu'elle s'est développée, a généré des recompositions du jeu et de l'interdépendance (Filippi, 2002) des acteurs du contrat. Plusieurs producteurs énoncent spontanément que la négociation des engagements collectifs par leurs représentants et leur traduction pratique a renforcé leur savoir-faire organisationnel et leur noyau identitaire. Cette transformation des rapports coopérative-industriel mène progressivement les associations et leurs membres agriculteurs à l'autonomie, donc à leur "émancipation " (Constance, 2008). Ces apprentissages se concrétisent progressivement en savoir-faire: des actions innovatrices, des dynamiques de gouvernance des associations et de la coopérative, y compris à partir d'initiatives individuelles. Mais, comment prendre en compte les intérêts de ceux qui sont encore peu impliqués activement (Dulcire et Roche, 2007), mais qui doivent respecter les règles collectives, sous peine d'exclusion de leur association?

\section{Du mythe à des pratiques codifiées : une incompréhension initiale}

Les producteurs expriment tous en cours d'entretien leur difficulté à comprendre au début les termes du contrat qu'ils acceptent alors et, donc, à les subir. La demande industrielle de qualité les a obligés à s'accorder sur un travail dont le contrat évalue la qualité par des critères tels que : originalité, procédés pré- et postrécolte, tradition, goût... (Dulcire, 2005) - travail que Hubert (2001) qualifie de " réactivation du local".

Nous pouvons considérer cette requalification régionale comme une renaissance, une nouvelle "sensibilité à l'égard d'un béritage [...] collectif dont la reconnaissance, la conservation et la transmission [sont] problématique[s]" (Tornatore, 2007). Cet ancrage territorial du cacao "nacional", sa typicité, s'est donc fondé sur son histoire, mais aussi sur une reconstruction de l'identité des producteurs, ce que Hillier et al. (2004) désignent comme " une transformation des rapports sociaux et de gouvernance". Ce processus a constitué un changement vers une construction technique et organisationnelle du " local ", sa " réinvention" (Zimmermann, 2005), un lieu de construction d'identités et d'actions des agriculteurs, du collectif et de l'industriel. Ces modifications progressives sont ici "l'objet même du processus transformateur" (Foudriat et Immel, 2003), inscrit dans le contrat originel qui est progressivement amendé en commun.

Cette démarche participative constitue dès le début un enjeu crucial pour une coopération durable entre les acteurs de ce contrat. Elle pose aussi la question du rôle d'un cadre préétabli comme celui du "bio-équitable " pour la construction d'un projet commun réaliste entre l'entreprise impliquée et les producteurs, d'une "représentation partagée permettant des décisions plus consensuelles et plus pertinentes" (Lévy, 2005).

\section{Et le chercheur expert}

\section{en cacao aromatique?}

Le chercheur joue différents rôles : porteparole de l'industriel ; traducteur entre deux mondes (Callon, 2003), entre l'industriel et le rural, et entre la France et l'Équateur ; et, enfin, médiateur entre ces deux acteurs. Mais il accompagne aussi comme expert l'évolution des systèmes techniques et l'organisation des producteurs.

Le chercheur a formalisé des liens avec les organisations de producteurs et l'industriel pour construire ensemble des "objectifs partagés " (Torre et Chia, 2001). Il a été commandité pour satisfaire les deux parties, par la prise en compte de leurs enjeux spécifiques, et se trouve ainsi écartelé entre ordonnateur et exécutant. Il est donc amené à se demander si sa pratique porte autant sur les obstacles concrets se dressant entre et devant ces personnes accompagnées que sur le "développement de leurs capacités d'adaptation " (Vallerie et Le Bossé, 2003).
Ses activités, en réponse à la demande des acteurs, comme expert, mais aussi comme intermédiaire, illustrent alors l'ambiguité des fonctions sollicitées au regard de son métier de chercheur. Sa posture mais aussi son savoir-faire, son engagement à répondre aux acteurs, expliquent-ils de façon effective la construction du système relationnel? La confiance est une condition indispensable au fonctionnement d'un dispositif participatif et l'homme concerné a pris le pas sur le chercheur impliqué. Certains producteurs expriment que leur travail avec le chercheur face aux exigences de l'acheteur les a aidés à surmonter les contraintes et à se convaincre qu'il y avait des améliorations possibles. Cet engagement progressif après le démarrage se matérialise par des initiatives, des propositions discutées pour leur intégration concrète dans le contrat, lequel devient alors un objet commun.

\section{De la négociation passive à l'autonomie des producteurs?}

Ce processus de recherche participative s'est construit en réponse à une demande industrielle. La recherche a été le médiateur de celle-ci, le " chef d'orchestre " d'un système d'acteurs - industriel et producteurs organisés - et de points de vue et d'intérêts différents, ce que Damon (2002) appelle le "jeu de co".

Le changement technique et organisationnel a été imposé à la coopérative par l'industriel, à partir d'une culture historique et identitaire. Les producteurs ont au début accepté et respecté passivement les exigences pour bénéficier des incitations matérielles accordées (tableau 1). Puis, la concertation enclenchée a débouché peu à peu sur des ajustements communs du cahier des charges, qui augmentent la stabilité des relations entre l'industriel et les agriculteurs. Les différents résultats positifs, énoncés de façon plus ou moins directe par les acteurs, attestent de cette convergence progressive, ce que nous pourrions appeler la construction sociale d'un objet cacao de qualité (Callon, 2003). Cette confiance réciproque constitue un élément fondamental de la durabilité de la filière, dont l'organisation reste fragile : cette convergence progressive repose d'abord sur un simple ralliement des producteurs à leurs collègues innovateurs. 


\section{En conclusion :}

\section{des apprentissages à consolider}

Cette étude souligne la difficulté d'acteurs économiquement dominés à s'organiser collectivement en réponse à une demande, à une opportunité, S'organiser n'a pas été dans ce cas un choix individuel mais une obligation extérieure contraignante. Les hésitations et les doutes exprimés, en particulier par les producteurs, concernent ici les nouvelles façons de faire, techniques et surtout organisationnelles. Ils sont largement issus du paradoxe de l'innovation d'un cacao typé conduite avec le chercheur, en réponse à une demande industrielle, qui oblige à la renaissance de leur cacao oublié.

Les producteurs de ce nouveau cacao ne sont pas encore devenus autonomes et montrent leur appréhension face au "challenge de demain" (Dulcire et Roche, 2008), à leur nouveau métier ou encore au futur générationnel. Leur gestion du contrat est un apprentissage, qui améliore aussi leurs capacités d'adaptation individuelles et collectives aux évolutions des contextes.

\section{Remerciements}

Je remercie Eduardo Chia pour les discussions qui ont permis d'avancer sur le fond et sur la forme de cet article, ainsi que les évaluateurs de la revue qui m’ont permis de l'améliorer.

\section{Références}

Akrich M. La construction d'un système sociotechnique, esquisse pour une anthropologie des techniques. In : Akrich M, Callon M, Latour $\mathrm{B}$, eds. Sociologie de la traduction. Paris: les presses Mines, 2006.

Callon M. Science et société : les trois traductions. Les cahiers du MUR.S 2003 ; 42 : 55-69.

Constance $\mathrm{DH}$. The emancipatory question: the next step in the sociology of agrifood systems? Agric Hum Values 2008 ; 25 : 151-5.

Damon L. La dictature du partenariat. Vers de nouveaux modes de management public ? Futuribles $2002 ; 273$ : 27-41.

Denoux $\mathrm{P}$. Un objet inter-culturel entre polémique et polysémique. Actes de la $12^{\mathrm{e}}$ Université de l'innovation rurale " Territoires ruraux, Comment débattre des sujets qui fâchent? ", Marciac, 2006.

Dulcire M. Une culture patrimoniale du mythe à la renaissance, le café "Bonifieur " de Guadeloupe (FWI). Anthropology of Food 2005; (4). www.aofood.org,

Dulcire M, Roche G. Sistema de toma de decisión y aprendizajes de los agricultores. El caso del sector de cacao orgánico en Sao Tomé. IX Congreso de Sociología Poder, cultura y civilización; sociología de la alimentación, Barcelona, 2008.

Dulcire M, Roche G. Chercheurs - agriculteurs industriel : co-construction d'une filière de cacao fin et "bio " en Equateur. Troisième conférence Living Knowledge, "Quand chercheurs et citoyens coproduisent les savoirs et les décisions scientifiques et techniques ", Paris, 2007.

Faure G, Hocdé H, Meneses D. Les organisations paysannes du Costa Rica construisent leur vision de I'agriculture familiale: une démarche de recherche-action marquée par une rupture. Cah Agric 2007; 16: 205-11. DOI: 10.1684/ agr.2007.0094.

Filippi M. Les sociétés coopératives agricoles entre ancrage territorial et intégration économique: éléments de méthodologie. Etudes et Recherches $2002 ; 33$ : 79-94.
Fixmer $P$ Brassac $C$. La décision collective comme processus de construction de sens. In Bonardi C, Grégori N, Ménard JY, Roussiau N, eds. Psychologie sociale appliquée. Emploi, travail, ressources humaines. Paris: InPress, 2004.

Freire P. Pédagogie des opprimés. Paris: Maspero, 1974.

Hillier J, Moulaert F, Nussbaumer J. Trois essais sur le rôle de l'innovation sociale dans le développement territorial. Géographie, Economie. Société 2004 ; 6 : 129-52.

Hubert A. Systèmes agroalimentaires localisés, réflexions d'une anthropologue. In: Systèmes agroalimentaires localisés: terroirs, savoir faire, innovations. Montpellier: Inra-Cirad-Cnearc, 2001.

Kaufman JC. L'entretien compréhensif. Paris: Armand Colin, 2005.

Lévy A, De. I'histoire à l'actualité de la psychosociologie. Sciences de I'Homme \& Sociétés 2005 $79: 12-6$.

Salette J. La typicité : une notion nouvelle au service du produit, de ceux qui l'élaborent, et de ceux qui le consomment en I'appréciant. Revue des Enologues 1997 ; 85 : 11-3.

Thill G. Le dialogue des savoirs. Les réseaux associatifs, outils de croisement entre la science et la vie. Bruxelles : Charles Léopold Mayer, 2001.

Tornatore JL. Les formes d'engagement dans l'activité patrimoniale, de quelques manières de s'accommoder au passé. In: Meyer V, Walter J, eds. Formes de l'engagement et espace public, questions de communication. Nancy: Presses Universitaires, 2007.

Torre A, Chia E. Pilotage d'une AOC fondée sur la confiance. Le cas de la production de fromage de Comté. Gérer et comprendre 2001 ; 65 : 55-67.

Vallerie B, Le Bossé Y. Le développement du pouvoir d'agir des personnes et des collectivités. Sauvegarde de l'enfance 2003 ; 58 : 144-55.

Wyvekens A. De «Whats Works" en "bonnes pratiques ". Y a-t-il un bon usage du pragmatisme anglo-saxon? Les cahiers de la sécurité intérieure 2003 ; 51 : 7-19.

Zimmermann JB. Entreprises et territoires: entre nomadisme et ancrage territorial. Revue de I'Ires $2005 ; 47: 21-36$. 